Positionspapier

\title{
Gesundheitsförderung in der Arbeitswelt
}

\author{
Arbeitsgruppe 2 „Betriebliche Gesundheitsförderung“ \\ des Deutschen Forums Prävention und Gesundheitsförderung
}

(Stand: 1. Februar 2007)

\section{Ausgangslage}

Die Arbeitsgruppe 2 (AG 2) „Betriebliche Gesundheitsförderung“(BGF) des Deutschen Forums Prävention und Gesundheitsförderung ist ein Zusammenschluss der wichtigsten Akteure aus dem Bereich der BGF. Sie arbeitet auch für die Initiative Neue Qualität der Arbeit (INQA) und ist Beirat für das Deutsche Netzwerk Betriebliche Gesundheitsförderung (DNBGF).

Die AG 2 wird mit Unterstützung der Bundesanstalt für Arbeitsschutz und Arbeitsmedizin (BAuA) durch das Bundesministerium für Arbeit und Soziales (BMAS) geleitet. Mitglieder sind neben dem BMAS und dem Bundesministerium für Gesundheit (BMG), die Spitzenverbände der Krankenkassen und der Unfallversicherungsträger, die Arbeitsschutzbehörden des Bundes und der Länder, die Verbände der Sozialpartner, die Deutsche Rentenversicherung Bund, die Verbände der Betriebs- und Werksärzte und der Sicherheitsingenieure, sowie ausgewählte Fachverbände z. B. aus der Weiterbildung und Stiftungen. Die AG 2 versteht sich als Plattform zum Informationsaustausch und zur Förderung der Umsetzung gesundheitsfördernder Aktivitäten im Betrieb.

In ihrer Arbeit orientiert sich die AG 2 an den Empfehlungen der Expertenkommission „Betriebliche Gesundheitspolitik“ (Bertelsmann Stiftung/Hans-Böckler-Stiftung 2004, Zukunftsfähige Betriebliche Gesundheitspolitik) sowie an europäischen und internationalen Empfehlungen zu Prävention und Gesundheitsförderung in der Arbeitswelt. 


\section{Herausforderung}

Gewandelte Rahmenbedingungen - geprägt durch Globalisierung, Strukturwandel in Wirtschaft und Verwaltungen, demografische Entwicklung und gewandeltes Krankheitspanorama - stellen große Herausforderungen dar für den Wirtschaftsstandort Deutschland sowie die Menschen, die hier leben und arbeiten. Sie erfordern eine Aufwertung und Weiterentwicklung der betrieblichen Gesundheitspolitik auf allen Ebenen. Zu diesem Schluss kommt die Expertenkommission „Betriebliche Gesundheitspolitik" der Bertelsmann Stiftung und der Hans-Böckler-Stiftung und hat dazu im Rahmen ihrer zweijährigen Arbeit eine langfristige Strategie entwickelt sowie Empfehlungen an die Unternehmen, Sozialpartner, die überbetrieblichen Akteure in den sozialen Sicherungssystemen und den Staat formuliert (Bertelsmann Stiftung/HansBöckler-Stiftung, 2004).

Um die notwendige Anpassung an den Wandel von Arbeit und Gesellschaft zu leisten, setzt die EU-Kommission in einer neuen Gemeinschaftsstrategie für Gesundheit und Sicherheit am Arbeitsplatz 2002-2006 auf das globale Konzept des „Wohlbefindens bei der Arbeit“ und zielt auf eine Verbesserung der „Qualität der Arbeit“ und den „Aufbau von Partnerschaften zwischen allen Akteuren im Bereich Gesundheit und Sicherheit“ (EU-Kommission 2002).

Vision der betrieblichen Gesundheitspolitik ist „gesunde Arbeit in gesunden Organisationen“. Bei der Bearbeitung gesundheitsrelevanter Problemstellungen sind nicht mehr allein die Arbeitsbedingungen und die Mensch-Maschine-Schnittstelle zu betrachten, sondern die gesamte Organisation und die Mensch-Mensch-Schnittstelle ist in den Blick zu nehmen. Zu verbessern gilt es insbesondere die Qualität der Menschenführung und die Qualität der zwischenmenschlichen Beziehungen und die Unternehmenskultur. „Das Hauptgewicht sollte bei der Verhütung gesundheitlicher Probleme liegen und nicht bei ihrer nachgehenden Bewältigung. Gesundheitsförderung und Prävention müssen als Führungsaufgabe wahrgenommen und nicht nur von nachgeordneten Fachabteilungen bearbeitet werden. Betriebliche Gesundheitspolitik muss unter Einbeziehung der Betroffenen praktiziert und nicht nur „Top-down“ verordnet werden. Und sie muss in ihrer Ausgestaltung vielfältig sein, d.h. den unterschiedlichen Bedürfnissen einzelner Branchen und Betriebsgrößen entsprechen.“ (Bertelsmann Stiftung/Hans-Böckler-Stiftung 2004, S. 21). 
Die Umsetzung einer vorausschauenden, alters- und geschlechtssensiblen betrieblichen Gesundheitspolitik liegt in der Verantwortung der Betriebe. Unternehmen, Verwaltungen und Dienstleistungsorganisationen sollten zukünftig selbst mehr in das Wohlbefinden und die Gesundheit der Mitarbeiter investieren: zuallererst aus sozialer Verantwortung gegenüber den Beschäftigten, aber auch wegen der dadurch zu erzielenden höheren Wettbewerbsfähigkeit, d.h. aus eigenem wirtschaftlichen Interesse, sowie zur Entlastung der sozialen Sicherungssysteme, was den Unternehmen selbst wiederum in Form niedrigerer Lohnnebenkosten zugute kommt.

Umsetzen lässt sich eine wirksame betriebliche Gesundheitspolitik durch die konsequente Verfolgung gesetzlicher Zielvorgaben des Arbeits- und Gesundheitsschutzes, durch Projekte der betrieblichen Gesundheitsförderung und durch die Etablierung eines in die Routinen integrierten betrieblichen Gesundheitsmanagements.

Die Beschäftigten sollten bei der Planung und Umsetzung aller Aktivitäten aktiv beteiligt werden. Und sie sollten darüber hinaus befähigt werden, durch eine gesundheitsbewusste Arbeits- und Lebensweise die eigene Gesundheit zu pflegen und zu erhalten.

Die AG 2 des Deutschen Forums Prävention und Gesundheitsförderung schließt sich den vorangegangenen Überlegungen und Vorschlägen an. Sie baut in ihren Zielen, Arbeitsschwerpunkten und Aktivitäten darauf auf, um einer aktiven und nachhaltigen betrieblichen Gesundheitspolitik zum Durchbruch zu verhelfen. Dabei leistet sie einen wichtigen Beitrag zum Erhalt und zur Förderung der Arbeits- und Beschäftigungsfähigkeit von Arbeitnehmern und damit zur Wettbewerbsfähigkeit von Unternehmen und Organisationen.

\section{Ziele der Arbeitsgruppe}

In ihren o. g. Funktionen übernimmt die Arbeitsgruppe insbesondere folgende Aufgaben:

- $\quad$ Entwicklung von Empfehlungen für nationale Ziele im Bereich der betrieblichen Prävention und der betrieblichen Gesundheitsförderung. 
- Formulierung vordringlicher Aufgabenfelder für betriebliche Prävention und betriebliche Gesundheitsförderung.

- Impulsgeber und Berater insbesondere für INQA, das Deutsche Forum Prävention und Gesundheitsförderung sowie das DNBGF.

- $\quad$ Förderung der Umsetzung von Beispielen guter Praxis im Bereich betrieblicher Prävention und betrieblicher Gesundheitsförderung.

- $\quad$ Förderung der Wirksamkeit von Maßnahmen der betrieblichen Prävention und betrieblichen Gesundheitsförderung durch Standardisierung.

- Förderung des Erfahrungsaustausches und der Kooperation der überbetrieblichen Akteure sowie der Festlegung von Gesundheitszielen auf Bundes- und Länderebene.

\section{Arbeitsschwerpunkte der AG 2}

Zur Förderung von Gesundheit und Wohlbefinden bei der Arbeit setzt sich die Arbeitsgruppe folgende Ziele und widmet sich bei der Zielerreichung den dazugehörigen Arbeitsschwerpunkten bei denen soweit sinnvoll auch Genderaspekte mit einfließen:

1. Entwicklung und Förderung von Konzepten eines umfassenden Programms zur Unterstützung einer sozialen Umgebung (Unternehmenskultur) für die Beschäftigten und einer gesunden Organisation/Arbeitsumwelt.

Federführung: Kowalski, Heinz; Institut für Betriebliche Gesundheitsförderung BGF GmbH, Köln

- Entwicklung eines Methodeninventars zur Selbstbewertung von Unternehmen und Verwaltungen.

- Darstellung und Förderung von Beispielen guter Praxis.

- Qualifizierungsmaßnahmen für Führungskräfte und Personalentwickler.

2. Förderung der psychischen Gesundheit bei der Arbeit

Federführung: Breithaupt, Rainer; Bundesministerium des Innern, Berlin

- Gestaltung gesundheitsförderlicher Arbeitsbedingungen. 
- $\quad$ Förderung von Konzepten zur Stärkung der sozialen Kompetenzen und Kommunikationsfähigkeiten insbesondere der betrieblichen Führungsebene zur Unterstützung von fairen Kommunikationsprozessen.

- Sensibilisierung für die Verbesserung der Akzeptanz der psychischen/ emotionalen Gesundheit.

- Sichtbar machen von psychischen Fehlbelastungen und ihrer Folgen.

- Verbreitung von Informationen zur Vermeidung psychischer Fehlbelastungen und zur Früherkennung möglicher psychischer Krankheiten.

3. Förderung der individuellen gesundheitlichen Kompetenz für eine nachhaltige Beschäftigungsfähigkeit

Federführung: Dr. Bonitz, Dieter AOK Bundesverband, Bonn

4. Förderung einer alternsgerechten Gestaltung der Arbeit Federführung: Wolters, Jürgen; BKK Bundesverband, Essen

5 Förderung der Umsetzung der BGF in KMU und für Selbstständige Federführung: Pelster, Klaus; Institut für Betriebliche Gesundheitsförderung BGF GmbH, Köln

- Unterstützung von Netzwerken.

6. Stärkung der Prävention und Gesundheitsförderung für Risikogruppen (z.B. Baugewerbe, Pflegeberufe, Lehrerinnen und Lehrer, Ver- und Entsorgung) Federführung: Teske, Ulrike; ver.di bundesverwaltung, Berlin Pelster, Klaus; Institut für Betriebliche Gesundheitsförderung BGF GmbH, Köln

- $\quad$ Analyse struktureller Hemmnisse und Empfehlungen zu ihrer Überwindung in der Praxis.

Die Mitglieder der Arbeitsgruppe wirken mit ihren Institutionen aktiv an der Umsetzung der Ziele der BGF in Deutschland mit: Durch die Beteiligung an gemeinsamen Veranstaltungen, Öffentlichkeitsarbeit, Information der AG 2 über aktuelle Projekte und Vorhaben sowie die Entwicklung und Umsetzung gemeinsamer Ziele, Strategien und Aktivitäten. 
Alle zwei Jahre wird ein zusammenfassender Bericht über die Aktivitäten der AG 2 erstellt und dem Plenum des Deutschen Forum Prävention und Gesundheitsförderung zur Verfügung gestellt. 